\title{
Photoelectron spectroscopy investigations of recombination contacts for tandem organic solar cells
}

\author{
Selina Olthof, ${ }^{\text {a) }}$ Ronny Timmreck, Moritz Riede, and Karl Leo \\ Institut für Angewandte Photophysik, Technische Universität Dresden, Dresden 01062, Germany
}

(Received 15 December 2011; accepted 20 February 2012; published online 14 March 2012)

\begin{abstract}
Recombination contacts play an important role in highly efficient organic tandem solar cells. We present a photoelectron spectroscopy study on contact systems that have previously been shown to work efficiently as recombination contacts. Here, the conversion of an electron current into a hole current is realized either by insertion of gold clusters or by a highly doped pn-junction. From the measured energy level alignments, we show that the working principles of these two approaches are significantly different. For gold clusters, the recombination current is promoted by an accumulation of charge carriers, while for doped pn-junctions, it is achieved by tunneling through a depletion layer. (C) 2012 American Institute of Physics. [http://dx.doi.org/10.1063/1.3693385]
\end{abstract}

Organic solar cells are promising candidates for flexible, low cost, and large area photovoltaic applications. Even though organic molecules have large absorption coefficients, they often only absorb in a narrow energy band which limits the solar cell performance. To circumvent this disadvantage, it is possible to combine two or more cells consisting of different absorbers into a single device forming a tandem cell. $^{1,2}$ If an ideal connection recombination contact between the cells is realized, the open circuit voltage $\left(\mathrm{V}_{\text {oc }}\right)$ of the tandem cell equals the sum of the $\mathrm{V}_{\mathrm{oc}}$ of the single cells. Such a recombination contact has to convert the electron current from the first solar cell into a hole current for the second solar cell and should meet two requirements: (1) under illumination, the splitting of the Fermi level at this interface has to be avoided, so no reverse voltage is produced ${ }^{3,4}$ and (2) an efficient tunneling between electron and hole states at the interface has to be provided for. A common way to realize a recombination contact is the insertion of metal clusters or thin metal layers at the interface. ${ }^{5,6}$ In this case, the metal islands quench the excitons, remove the Fermi level splitting, and introduce a density of states (DOS) that reaches up to the Fermi energy providing states for an electron-hole recombination. Another concept is the use of highly doped layers to form a pn junction between the two sub-cells. ${ }^{3,7}$ Such an interface is a suitable recombination contact as the high concentration of free charge carriers moves the lowest occupied molecular orbital (LUMO) of the acceptor and highest unoccupied molecular orbital (HOMO) of the donor close to the Fermi level and thereby reduces the energetic barrier, while the thin depletion regions can easily be tunneled through. ${ }^{8}$

In this letter, we investigate the energy level alignment of a commonly used donor acceptor interface consisting of $\mathrm{N}, \mathrm{N}, \mathrm{N}^{\prime}, \mathrm{N}^{\prime}$-tetrakis (4-methoxyphenyl)-benzidine (MeO-TPD) and $\mathrm{C}_{60}$ by UV photoelectron spectroscopy (UPS). We previously studied and published prototype tandem devices employing the same recombination contacts as under investi-

\footnotetext{
a) Author to whom correspondence should be addressed. Electronic mail: solthof@princeton.edu. Present address: Department of Electrical Engineering, Princeton University, New Jersey 08544, USA.
}

gation here. ${ }^{3}$ A summary of the characteristic values of these single and tandem cells is reproduced in Table I. This current work is supposed to complement these device studies and provide an understanding of the working principle and design requirements of recombination contacts.

The organic layers are prepared by thermal evaporation under ultra high vacuum. Details about the experimental setup can be found elsewhere. ${ }^{9}$ The materials MeO-TPD, 2,3,5,6-tetrafluoro-7,7,8,8-tetracyanoquinodimethan $\quad\left(\mathrm{F}_{4^{-}}\right.$ TCNQ), $\mathrm{C}_{60}$, and acridine orange base (AOB) are deposited from heated crucibles. Doping is achieved by co-evaporating host and dopant, while the evaporation rates are controlled independently by two quartz crystals. As substrate, a sputter cleaned silver foil (purity 99.995\%) is used, covered by $30 \mathrm{~nm}$ of freshly evaporated gold.

First, the interface between the intrinsic layers of $\mathrm{C}_{60}$ and MeO-TPD is characterized in order to compare the alignment to the cases when the metal clusters or dopants are introduced. In Table I, this interface equals the one used in tandem cell 1 . It can be seen from the $\mathrm{V}_{\mathrm{oc}}$ that such an interface does not act as a recombination contact when implemented into a device, but rather as a backward solar cell, preventing an increase of $\mathrm{V}_{\mathrm{oc}}$ compared to the single cell device. For the UPS measurements, a gold substrate is used instead of the device relevant indium tin oxide (ITO), as ITO often shows degradation under UV illumination. ${ }^{10}$ The substrate is covered by $10 \mathrm{~nm}$ of p-doped MeO-TPD; the doping

TABLE I. Characteristic values of a single solar cell (stack: ITO/p-MeO$\mathrm{TPD} / \mathrm{MeO}-\mathrm{TPD} / \mathrm{C}_{60} / \mathrm{BPhen} / \mathrm{Al}$ ) and the tandem solar cells that exploit the recombination contacts investigated in this paper. The structure of the tandem cells is ITO/p-MeO-TPD/C $60 / \mathrm{x} / \mathrm{MeO}-\mathrm{TPD} / \mathrm{C}_{60} / \mathrm{BPhen} / \mathrm{Al}$, where $\mathrm{x}$ stands for the different recombination contacts. The loss of $\mathrm{V}_{\mathrm{oc}}$ is the percentage missing for a full doubling of $\mathrm{V}_{\mathrm{oc}}$. Values are taken from our previous publication of Timmreck et $a l^{3}$

\begin{tabular}{lcccc}
\hline \hline & Interlayer x & $\mathrm{V}_{\mathrm{oc}}(\mathrm{eV})$ & Loss of $\mathrm{V}_{\mathrm{oc}}(\%)$ & $\mathrm{FF}(\%)$ \\
\hline Single cell & - & 0.53 & - & 50 \\
Tandem cell 1 & none & 0.57 & 46.2 & 39 \\
Tandem cell 2 & 1 nm gold & 0.96 & 9.4 & 53 \\
Tandem cell 3 & pn junction & 1.04 & 1.9 & 48 \\
\hline \hline
\end{tabular}


(a)

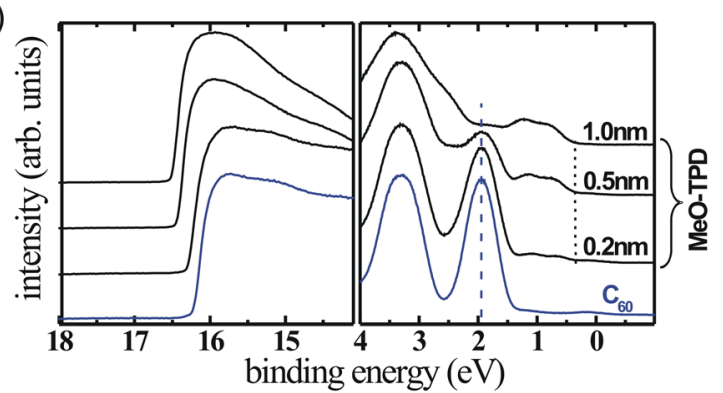

(b)

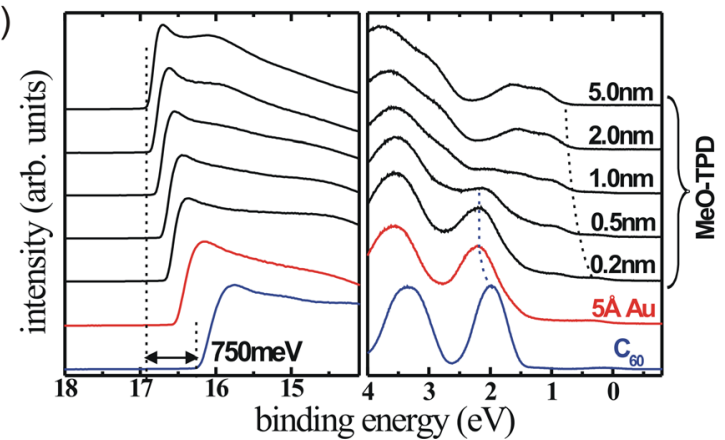

(c)

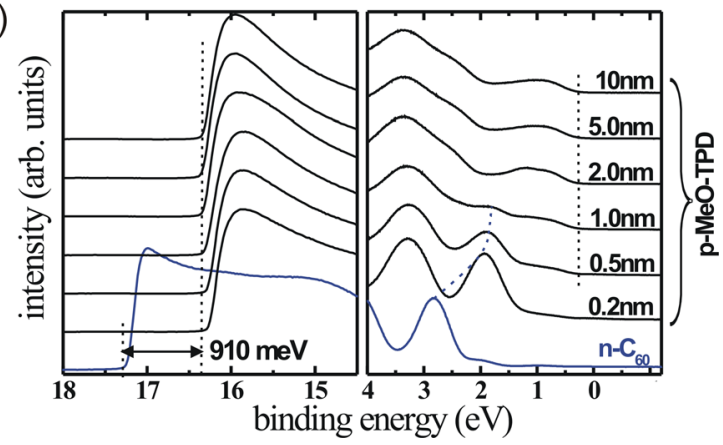

FIG. 1. (Color online) UPS measurements of the interfaces between layers of (a) intrinsic $\mathrm{C}_{60}$ and subsequently deposited MeO-TPD. (b) $\mathrm{C}_{60}$ and MeO-TPD with $0.5 \mathrm{~nm}$ of gold deposited in between and (c) $\mathrm{C}_{60}$ doped by AOB $(20 \mathrm{~mol} \%)$ and MeO-TPD doped by $\mathrm{F}_{4}$-TCNQ (40 mol \%). The dotted lines mark the change in high binding energy cutoff, $\mathrm{C}_{60}$ peak position, and MeO-TPD HOMO cutoff.

ensures an independence of the energetic alignment on the substrate work function ${ }^{11}$ and leads to an equal energetic alignment of the following layers compared to the previously reported tandem devices given in Table I. Additional intrinsic layers can be omitted during the investigation as they have no or very little effect on the alignment. ${ }^{9}$ On top of this p-doped layer, $8 \mathrm{~nm}$ of $\mathrm{C}_{60}$ are evaporated and measured by UPS shown as the bottom curve in Fig. 1(a). The ionization potential (IP) is $6.46 \mathrm{eV}$ and the distance from the HOMO onset to the Fermi energy is $1.45 \mathrm{eV}$ as can be seen in the resulting energy level alignment in Fig. 2(a). Assuming an electron affinity of $4 \mathrm{eV}$ (Ref. 12) and, therefore, a transport gap of $2.46 \mathrm{eV}$, the distance between the Fermi energy and the LUMO is about $1 \mathrm{eV}$. The measurement is carried on by incrementally depositing the MeO-TPD interface in sub $\mathrm{nm}$ steps on top while for every thickness an UPS spectrum is recorded as shown in Fig. 1(a). Since the work function of the $\mathrm{C}_{60}$ layer is close to the IP of the MeO-TPD, a gradual change of $300 \mathrm{meV}$ is observed in the vacuum level that moves the MeO-TPD HOMO away from the Fermi energy. However, beginning from $1 \mathrm{~nm}$ thickness, the sample shows charging, which expresses itself in a shift of several tenths of meV of the whole spectra during repeated measurements. This is expected, as the deeper lying HOMO of $\mathrm{C}_{60}$ forms a barrier for the holes generated by the removal of the photoelectrons during measurement. The fact that this already happens at very low coverage gives first insight on the blocking behavior of this contact and suggests that this is indeed not a recombination contact. The offset between the LUMO of $\mathrm{C}_{60}$ and the HOMO of MeO-TPD at this interface is $1.36 \mathrm{eV}$, while the open circuit voltage measured for this interface in a solar cell is $\mathrm{V}_{\mathrm{oc}}=0.53 \mathrm{eV}$ (see Table I) and, therefore, much smaller. This discrepancy has been observed often and is due to contributions from the exciton binding energy ${ }^{13}$ as well as intermolecular charge-transfer states ${ }^{14}$ and disorder induced tail states. ${ }^{11,15,16}$

By repeating the measurement with a similar stack, but this time inserting a thin gold layer at the interface between $\mathrm{C}_{60}$ and MeO-TPD, the alignment is significantly modified. This interface used in tandem cell 2 in Table I was found to be a well working recombination contact with a loss of $\mathrm{V}_{\mathrm{oc}}$ of about $10 \%$ compared to a full doubling of the voltage. For the device, a $1 \mathrm{~nm}$ thick gold layer was used; however in the UPS investigations, this led to a suppression of the $\mathrm{C}_{60}$ features, therefore the thickness was reduced to $0.5 \mathrm{~nm}$. Such an interlayer leads to similar device performance $\left(\mathrm{V}_{\mathrm{oc}}=0.91 \mathrm{~V}\right.$ and $\mathrm{FF}=53 \%$ ). For the measurement of the energy level alignment, $\mathrm{C}_{60}$ is again evaporated on a gold substrate covered by p-doped MeO-TPD. The UPS measurement of this layer, shown as the bottom curve of Fig. 1(b), gives within

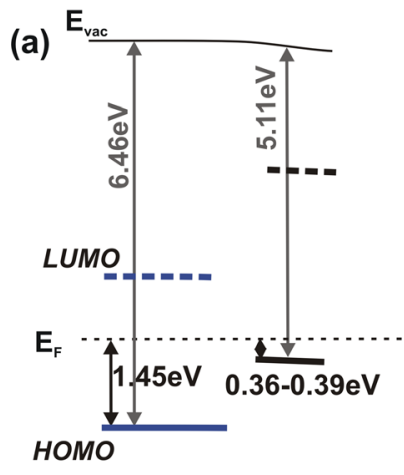

$\mathrm{C}_{60} \quad$ MeO-TPD (b)

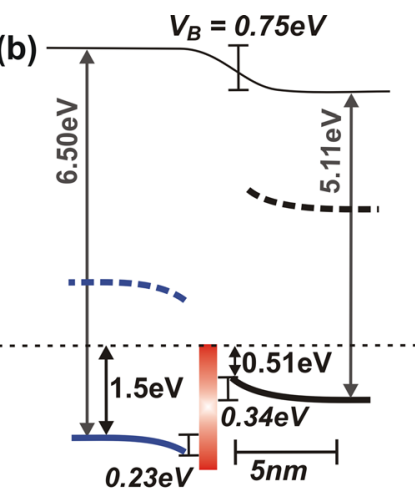

$\mathrm{C}_{60} \quad \mathrm{Au}$ MeO-TPD (c)

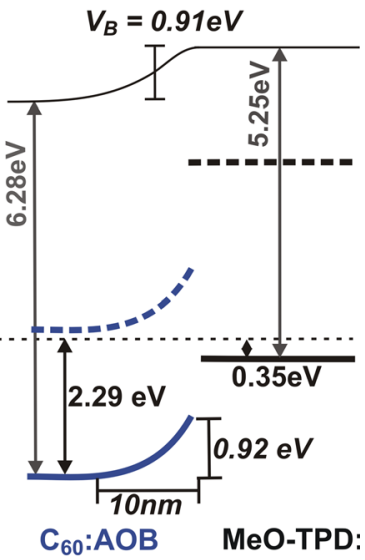

FIG. 2. (Color online) Schematic energy level alignment deduced from the measurements shown in Fig. 1: (a) alignment between intrinsic MeO-TPD and $\mathrm{C}_{60}$, (b) the same interface when $0.5 \mathrm{~nm}$ of gold are inserted, and (c) the highly doped pn junction. 
the experimental error the same alignment as in the previous sample. After the evaporation of $0.5 \mathrm{~nm}$ gold, the HOMO of $\mathrm{C}_{60}$ exhibits a significant shift of $230 \mathrm{meV}$ away from the Fermi energy, and the Fermi edge of the gold becomes visible. The shift is either due to the formation of an interface dipole upon contact with the gold or the penetrating gold clusters lead to an accumulation of electrons at the interface. The change in the HOMO position is accompanied by a downward shift of the vacuum level of $310 \mathrm{meV}$. As before, the MeO-TPD layer is incrementally built on top. Apparently, this layer is affected by the gold interlayer as well and shows a shifting of the HOMO position by $320 \mathrm{meV}$ with increasing layer thickness, indicating a transfer of electrons from the MeO-TPD to the gold at the interface. During the deposition, the $\mathrm{C}_{60}$ shows no further change in HOMO position, so the charge transfer is solely between the $\mathrm{MeO}$ TPD and Au. The measurement can only be continued until $5 \mathrm{~nm}$ MeO-TPD coverage, when again a charging of the sample is observed. We can conclude that the charge carrier conversion at the interface is much better than in case of the intrinsic interface, but still the process is not perfect, leading to an accumulation of photogenerated holes. The resulting energetic alignment is shown in Fig. 2(b). The total built-in potential of this interface, taken from the change in vacuum level position, is $\mathrm{V}_{\mathrm{B}}=0.75 \mathrm{eV}$. The offset between $\mathrm{C}_{60, \mathrm{LUMO}}$ and $\mathrm{MeO}-\mathrm{TPD}_{\text {Hомо }}$ is reduced by about $270 \mathrm{meV}$ compared to the intrinsic interface and in addition, there is a DOS reaching up to the Fermi energy due to the gold.

For the interface employing a pn junction, we use $\mathrm{F}_{4}$ TCNQ as the p-dopant in MeO-TPD at a high doping concentration of $40 \mathrm{~mol} \%$ and $\mathrm{AOB}$ as the n-dopant in $\mathrm{C}_{60}$ at $20 \mathrm{~mol} \%$, which was found to produce a very efficient recombination contact that nearly doubles the $\mathrm{V}_{\text {oc }}$ (see tandem cell 3 in Table I). For this sample, the n-doped $\mathrm{C}_{60}$ layer is directly put on the gold foil. The p-doped MeO-TPD can be omitted as the high doping concentration in $\mathrm{C}_{60}$ leads to Fermi level alignment of the film independent of the layers underneath. ${ }^{11}$ The UPS measurements are shown in Fig. 1(c). As expected, the energetic position of the $n$-doped $C_{60}$ differs from those in Figs. 1(a) and 1(b) as this time, the ndoping leads to an alignment, where the LUMO is only $170 \mathrm{meV}$ away from the Fermi energy. Upon the deposition of doped MeO-TPD, the $\mathrm{C}_{60}$ level shows a strong upward bending, typical for the formation of a depletion region due to charge transfer across the pn-junction. The HOMO of $\mathrm{C}_{60}$ can only be observed up to $0.5 \mathrm{~nm}$ MeO-TPD deposition. By then, it shows a level shifting of $920 \mathrm{meV}$. The HOMO of MeO-TPD shows no shift at all and remains $0.35 \mathrm{eV}$ below $\mathrm{E}_{\mathrm{F}}$. Apparently, the $40 \mathrm{~mol} \%$ doping by $\mathrm{F}_{4}-\mathrm{TCNQ}$ in $\mathrm{MeO}-$ TPD produces considerably more free charge carriers than the $20 \mathrm{~mol} \%$ doping of $\mathrm{C}_{60}$ by $\mathrm{AOB}$; therefore, the entire voltage drop takes place in $n-C_{60}$. The change in vacuum level position is $\mathrm{V}_{\mathrm{B}}=0.91 \mathrm{eV}$. The spectra do not show charging during the measurement done up to $10 \mathrm{~nm} \mathrm{MeO-}$ TPD thickness. However, from these measurements, we can only determine the amount of shifting and not the width of the depletion region in $\mathrm{n}-\mathrm{C}_{60}$; therefore, the measurement was repeated with reversed deposition sequence (data not shown). Just as before, the deposition of $\mathrm{n}-\mathrm{C}_{60}$ onto $\mathrm{p}-\mathrm{MeO}-$ TPD does not lead to a change in the MeO-TPD HOMO position. All the level bending takes place in the $\mathrm{C}_{60}$ which is found to take $10 \mathrm{~nm}$ to saturate. As can be seen in Fig. $2(\mathrm{c})$, the offset between $\mathrm{C}_{60, \mathrm{LUMO}}$ and $\mathrm{MeO}-\mathrm{TPD}_{\mathrm{HOMO}}$ is decreased to merely $0.52 \mathrm{eV}$. Furthermore, it has been reported that upon doping, the tailing states of the transport levels are pinned at the Fermi energy, ${ }^{11,15}$ so we can assume the tail of the DOS distribution reaches up to the $\mathrm{E}_{\mathrm{F}}$ on both sides of the $10 \mathrm{~nm}$ thick depletion layer.

Comparing the alignment of the two recombination contacts in Figs. 2(b) and 2(c) reveals that the effects causing an efficient recombination contact are rather different. In both cases, the distance between $\mathrm{C}_{60, \mathrm{LUMO}}$ and $\mathrm{MeO}-\mathrm{TPD}_{\mathrm{HOMO}}$ is reduced, and we observe states at or close to $\mathrm{E}_{\mathrm{F}}$ that prevent any Fermi level splitting under illumination which would create an unwanted backward $\mathrm{V}_{\mathrm{oc}}$. However, the sign of the built in voltage at the interface shows in opposite directions. In the case of the metal clusters, the $750 \mathrm{meV}$ field is created by a dipole formation and accumulation of charge carriers at the interface; in the case of the highly doped pn junction, the built in voltage of $910 \mathrm{meV}$ is created by the formation of a $10 \mathrm{~nm}$ wide depletion layer that the charge carriers tunnel through. Here, the advantage of using pn junctions over the metal recombination layers becomes obvious. Any sufficiently high doped material combination will be able to form a recombination contact by putting the transport levels in close proximity to the Fermi energy. However, in the case of the metals, it depends sensitively on the individual combination of organic material and metal whether the levels are shifted in the right direction and the built in field is of sufficient strength. This might explain why only few material combinations have so far been found that produce a doubling of the $\mathrm{V}_{\mathrm{oc}}$ when using the metal cluster approach, ${ }^{6,17}$ while most combinations suffer in some loss with regard to $2 \mathrm{~V}_{\mathrm{oc}}$.

In summary, we have presented photoelectron spectroscopy measurements of two different recombination contacts used in organic tandem solar cells employing either gold clusters or a highly doped pn junction. Comparing them to an interface without modification, we showed that both approaches reduce the tunneling distance for the recombination current between donor ${ }_{\text {HOMO }}$ and acceptor LUMO $_{\text {. Further- }}$ more, a density of states is appearing close to the Fermi energy promoting the tunneling current and preventing a Fermi level splitting under illumination. In both cases, a large built in field is created at the interface, but the direction of the field is opposed leading to a charge accumulation in case of the metal clusters and to the formation of a depletion region for the pn junction.

This work was supported by the BMBF within the projects OPEG (Grant No. 13N9720) and R2flex (Grant No.13N8855).

${ }^{1}$ J. Y. Kim, K. Lee, N. E. Coates, D. Moses, T.-Q. Nguyen, M. Dante, and A. J. Heeger, Science 317, 222 (2007).

${ }^{2}$ T. Ameri, G. Dennler, C. Lungenschmied, and C. J. Brabec, Energy Environ. Sci. 2, 347 (2009).

${ }^{3}$ R. Timmreck, S. Olthof, M. K. Riede, and K. Leo, J. Appl. Phys. 108, 033108 (2010).

${ }^{4} \mathrm{P}$. Wuerfel, Physics of Solar Cells, From Principles to New Concepts (Wiley-VCH, Weinheim, 2005).

${ }^{5}$ M. Hiramoto, M. Suezaki, and M. Yokoyama, Chem. Lett. 19, 327 (1990). 
${ }^{6}$ A. Yakimov and S. R. Forrest, Appl. Phys. Lett. 80, 1667 (2002).

${ }^{7}$ J. Drechsel, B. Maennig, F. Kozlowski, M. Pfeiffer, K. Leo, and H. Hoppe, Appl. Phys. Lett. 86, 244102 (2005).

${ }^{8}$ K. Harada, M. Riede, K. Leo, O. Hild, and C. M. Elliott, Phys. Rev. B 77, 195212 (2008).

${ }^{9}$ S. Olthof, R. Meerheim, M. Schober, and K. Leo, Phys. Rev. B 79, 245308 (2009).

${ }^{10}$ M. M. Beerbom, B. Laegel, A. J. Cascio, B. V. Doran, and R. Schlaf, J. Electron Spectrosc. Relat. Phenom. 152, 12 (2006).

${ }^{11}$ S. Olthof, W. Tress, R. Meerheim, B. Luessem, and K. Leo, J. Appl. Phys. 106, 103711 (2009).
${ }^{12}$ W. Zhao and A. Kahn, J. Appl. Phys. 105, 123711 (2009).

${ }^{13}$ P. I. Djurovich, E. I. Mayo, S. R. Forrest, and M. E. Thompson, Org. Electron. 10, 515 (2009).

${ }^{14}$ D. Veldman, S. Meskers, and R. Janssen, Adv. Funct. Mater. 19, 1939 (2009).

${ }^{15}$ M. Kroeger, S. Hamwi, J. Meyer, T. Riedl, W. Kowalsky, and A. Kahn, Org. Electron. 10, 932 (2009).

${ }^{16}$ W. L. Kalb, S. Haas, C. Krellner, T. Mathis, and B. Batlogg, Phys. Rev. B 81, 155315 (2010).

${ }^{17}$ G. Dennler, H. J. Prall, R. Koeppe, M. Egginger, R. Autengruber, and N. S. Sariciftci, Appl. Phys. Lett. 89, 073502 (2006). 